\title{
On the round-trip time for a photon propagating in the field of a plane gravitational wave
}

\author{
M Rakhmanov \\ Department of Physics and Astronomy, University of Texas at Brownsville, \\ Brownsville, TX 78520, USA \\ E-mail: malik.rakhmanov@utb.edu
}

\begin{abstract}
A network of large-scale laser interferometers is currently employed for searches of gravitational waves from various astrophysical sources. The frequency dependence of the dynamic response of these detectors introduces corrections to their antenna patterns which in principle can affect the outcome of the associated data-analysis algorithms. The magnitude of these corrections and the corresponding systematic errors have recently been estimated for searches of periodic and stochastic gravitational waves (2008 Class. Quantum Grav. 25 184017). However, the calculation of the detector response in that paper followed the traditional semi-rigorous approach which does not properly take into account the curved nature of spacetime. The question then arises as to whether the results will be the same if the calculation is done within the rigorous framework of general relativity. In this paper we provide such a derivation of the response of the detectors to gravitational waves. We obtain the photon propagation time from the solution of the equation for null geodesics and calculate the corresponding phase delay by solving the eikonal equation for curved spacetime. The calculations are then extended to include phase amplification from multi-beam interference in Fabry-Perot resonators which play an important role in the formation of the signal in these detectors.

PACS numbers: 04.80.Nn, 95.55.Ym
\end{abstract}

\section{Introduction}

Kilometer-scale gravitational wave detectors such as LIGO [1] and VIRGO 2] have recently reached their design sensitivities and have already established a number of astrophysical upper limits. The associated data-analysis efforts are currently focused on the development of algorithms for accurate waveform reconstruction and parameter estimation of the anticipated signal. The accuracy of these algorithms depends on the models which are utilized to describe the detector response to gravitational waves. In particular, the long-wavelength approximation [3] commonly practiced in such models introduces systematic errors in parameter estimation [4] which at first were thought to be large enough to modify the outcome of search algorithms, and even the reported upper limits. Recent studies, however, have shown that these errors were previously over-estimated; and, in fact, they do not significantly affect the search algorithms at the frequencies considered thus far [5. Curiously, in the course of these studies, it was realized that the usual derivations of the detector response (see, e.g. [6, 7, 8, 9, 10, 11, 12, and more recently [4, 5]) only partially follow the principles of 
general relativity. For example, in deriving the photon round-trip time, one usually integrates metric perturbations along the unperturbed photon trajectory, assuming that it is a straight line, and thus neglecting the slight bending of the trajectory due to the gravitational wave 13 . The calculations of the phase acquired by a photon in one round trip are also carried out assuming an idealized electromagnetic wave propagation, i.e. along the straight line. Such assumptions were also made in the recent analysis of the LIGO antenna patterns [14, 5]. In this paper we resolve these problems by providing a fully-relativistic analysis which properly takes into account the curved nature of the spacetime. We derive the photon propagation time by solving the equation for null geodesics. The round-trip phase is then found by solving the equation for eikonal in curved spacetime. The results agree with those obtained within the semi-rigorous approach.

\section{Equation for null geodesics}

Consider spacetime in which a plane-fronted gravitational wave is propagating on a flat background. Let the coordinates of this spacetime be $x^{\mu}=(c t, x, y, z)$ with Greek suffixes taking values $0,1,2,3$. Within the linearized theory, the metric of this spacetime is given by

$$
g_{\mu \nu}=\eta_{\mu \nu}+h_{\mu \nu}
$$

where $\eta_{\mu \nu}=\operatorname{diag}\{-1,1,1,1\}$ is the Minkowskii tensor and $h_{\mu \nu}$ is a small perturbation which represents the gravitational wave. In the transverse traceless gauge [15] the metric perturbation takes a particularly simple form:

$$
h_{\mu \nu}=\left[\begin{array}{cccc}
0 & & & \\
& h_{+} & h_{\times} & \\
& h_{\times} & -h_{+} & \\
& & & 0
\end{array}\right],
$$

where $h_{+}$and $h_{\times}$are functions of $c t+z$ only. Here we assumed that the gravitational wave is propagating in the negative $z$ direction and its principal axes of polarization are chosen along the $x$ and $y$ coordinates. We will also use two auxiliary coordinates,

$$
u=c t+z \quad \text { and } \quad v=c t-z,
$$

to simplify some of the following calculations. The fundamental form corresponding to metric (11) is given by

$$
d s^{2}=-d u d v+d x^{2}+d y^{2}+h_{+}(u)\left(d x^{2}-d y^{2}\right)+2 h_{\times}(u) d x d y .
$$

Propagation of light in this spacetime is described by a null geodesic: $x^{\mu}=x^{\mu}(\sigma)$, where $\sigma$ is the affine parameter along the curve. The tangent vector to this curve, which is also the photon momentum,

$$
p^{\mu}=\frac{d x^{\mu}}{d \sigma} \quad \text { and } \quad p_{\mu}=g_{\mu \nu} p^{\nu}
$$

satisfies the null condition:

$$
p_{\mu} p^{\mu}=0
$$

$\ddagger$ The analysis in this paper belongs to the linearized theory and therefore all the calculations are valid to first order in $h$ only. 
In the explicit form, the covariant components of the tangent vector are

$$
\begin{aligned}
& p_{v}=-\frac{1}{2} \frac{d u}{d \sigma}, \\
& p_{x}=\left[1+h_{+}(u)\right] \frac{d x}{d \sigma}+h_{\times}(u) \frac{d y}{d \sigma}, \\
& p_{y}=\left[1-h_{+}(u)\right] \frac{d y}{d \sigma}+h_{\times}(u) \frac{d x}{d \sigma}, \\
& p_{u}=-\frac{1}{2} \frac{d v}{d \sigma} .
\end{aligned}
$$

The geodesic equation can be written as

$$
\frac{d p_{\alpha}}{d \sigma}=\frac{1}{2} g_{\mu \nu, \alpha} p^{\mu} p^{\nu}
$$

Since the metric does not depend on the $x, y$, and $v$ coordinates, the corresponding components of the tangent vector are constant:

$$
\begin{aligned}
& p_{v}=p_{v 0}, \\
& p_{x}=p_{x 0}, \\
& p_{y}=p_{y 0} .
\end{aligned}
$$

The fourth equation is

$$
\frac{d p_{u}}{d \sigma}=\frac{1}{2}\left(p_{x}^{2}-p_{y}^{2}\right) h_{+}^{\prime}(u)+p_{x} p_{y} h_{\times}^{\prime}(u) .
$$

Its solution can be obtained directly from the null condition (6), and yields

$$
p_{u}(u)=\frac{1}{4 p_{v 0}}\left[p_{x 0}^{2}+p_{y 0}^{2}-\left(p_{x 0}^{2}-p_{y 0}^{2}\right) h_{+}(u)-2 p_{x 0} p_{y 0} h_{\times}(u)\right] .
$$

This completes the first integration of the geodesic equation.

Next, equation (12) together with the definition for $p_{v}$ in (7) can be integrated and yields

$$
u(\sigma)=u_{0}-2 p_{v 0} \sigma,
$$

where $u_{0}$ is the initial value for this coordinate. Equations (13) and (14) together with definitions (8) and (9) yield the solution for $x$ and $y$ :

$$
\begin{aligned}
& x(\sigma)=x_{0}+p_{x 0} \sigma\left[1-f_{+}(\sigma)\right]-p_{y 0} \sigma f_{\times}(\sigma), \\
& y(\sigma)=y_{0}+p_{y 0} \sigma\left[1+f_{+}(\sigma)\right]-p_{x 0} \sigma f_{\times}(\sigma),
\end{aligned}
$$

where $x_{0}$ and $y_{0}$ are the initial values for these coordinates, and $f_{i}$ are the averaged amplitudes of the gravitational wave:

$$
f_{i}(\sigma)=\frac{1}{\sigma} \int_{0}^{\sigma} h_{i}\left[u\left(\sigma^{\prime}\right)\right] d \sigma^{\prime}
$$

for $i=+, \times$. Finally, from (10) and (16) we obtain the solution for $v$ :

$$
v(\sigma)=v_{0}-2 \int_{0}^{\sigma} p_{u}\left[u\left(\sigma^{\prime}\right)\right] d \sigma^{\prime},
$$

where $v_{0}$ is the initial value for this coordinate. In the explicit form it is given by

$$
\begin{aligned}
v(\sigma)= & v_{0}-\frac{\sigma}{2 p_{v 0}}\left(p_{x 0}^{2}+p_{y 0}^{2}\right)- \\
& \frac{\sigma}{2 p_{v 0}}\left[\left(p_{x 0}^{2}-p_{y 0}^{2}\right) f_{+}(\sigma)+2 p_{x 0} p_{y 0} f_{\times}(\sigma)\right] .
\end{aligned}
$$

This completes the second integration of the geodesic equation. The arbitrary constants: $p_{v 0}, p_{x 0}, p_{y 0}, u_{0}, x_{0}, y_{0}$, and $v_{0}$ will be determined from the boundary conditions. 


\section{Round-trip propagation time}

\subsection{Boundary-value problem}

Consider a photon trajectory with fixed boundaries: $P_{0}=(0,0,0)$ and $P=(x, y, z)$, and assume that it starts at $P_{0}$ at time $t_{0}$ and ends at $P$ at time $t$. The trajectory is given by the null geodesic described in (17) 19) and (22) with the following initial values: $x_{0}=y_{0}=z_{0}=0$ and $u_{0}=v_{0}=c t_{0}$. The first three constants of integration can be found from (17), (18), and (19):

$$
\begin{aligned}
& p_{v 0}=-\frac{u-u_{0}}{2 \sigma}, \\
& p_{x 0}=\left(1+f_{+}\right) \frac{x}{\sigma}+f_{\times} \frac{y}{\sigma}, \\
& p_{y 0}=\left(1-f_{+}\right) \frac{y}{\sigma}+f_{\times} \frac{x}{\sigma} .
\end{aligned}
$$

In these equations $\sigma$ stands for the value of the affine parameter at the end point, $P$. Substituting (24) and (25) into (22), we obtain:

$$
v=c t_{0}-\frac{1}{2 p_{v 0} \sigma}\left[x^{2}\left(1+f_{+}\right)+y^{2}\left(1-f_{+}\right)+2 x y f_{\times}\right],
$$

in which we can replace $p_{v 0}$ from (23) with the result

$$
c^{2}\left(t-t_{0}\right)^{2}=r^{2}+\left(x^{2}-y^{2}\right) f_{+}+2 x y f_{\times} .
$$

Here we introduced the short-hand notation: $r \equiv \sqrt{x^{2}+y^{2}+z^{2}}$.

If upon rearching point $P$, the photon changes its course and proceeds along a different trajectory the solution can be found in a similar way. Consider for example the situation when the photon travels back to the point of origin. In other words, assume that the photon trajectory starts at $P$ at time $t_{1}$ and ends at $P_{0}$ at time $t$. In this case, the trajectory is also given by equations (17) 19) and (22) but with different boundary conditions. The initial values for the coordinates are now $c t_{1}, x, y, z$, whereas the final values are $x=y=z=0$ and $u=v=c t$. Consequently, the first three constants of integration are:

$$
\begin{aligned}
& q_{v 0}=-\frac{u-u_{0}}{2 \sigma}, \\
& q_{x 0}=-\left(1+g_{+}\right) \frac{x}{\sigma}-g_{\times} \frac{y}{\sigma}, \\
& q_{y 0}=-\left(1-g_{+}\right) \frac{y}{\sigma}-g_{\times} \frac{x}{\sigma},
\end{aligned}
$$

where $g_{i}$ are the averaged amplitudes of the gravitational wave, (20), for the return trip. Substituting equations (28), (29), and (30) into (22), we obtain

$$
c^{2}\left(t-t_{1}\right)^{2}=r^{2}+\left(x^{2}-y^{2}\right) g_{+}+2 x y g_{\times} .
$$

Equations (27) and (31) determine the duration for the forward and the return trips.

\subsection{Photon propagation time}

We can now apply the above formulae to calculate the round-trip time for a photon propagating between two inertial test masses. Assume that the points $P_{0}$ and $P$ correspond to the two test masses. Note that the coordinates of the test masses do not change under the influence of a gravitational wave [15, so that this physical situation 
indeed corresponds to the solution of the geodesic equation with fixed boundaries described above. Consider the equation for the forward propagation time (27). To first order in $h$, its solution is

$$
c\left(t-t_{0}\right)=r+\frac{1}{2 r}\left[\left(x^{2}-y^{2}\right) f_{+}+2 x y f_{\times}\right] .
$$

Let the unperturbed propagation time between the test masses be $T$, i.e. $T=r / c$. Then the duration of the forward trip in the presence of a gravitational wave is

$$
T_{1}(t)=T+\frac{1}{2} T\left[\left(a_{x}^{2}-a_{y}^{2}\right) f_{+}(t)+2 a_{x} a_{y} f_{\times}(t)\right]
$$

where $\hat{a} \equiv(x / r, y / r, z / r)$. Here we explicitly indicated that $f_{i}$ are functions of time. To find this functional dependence, we change the integration variable $\sigma^{\prime}$ in (20) to $u^{\prime}$, using (23):

$$
f_{i}(t)=\frac{1}{u-u_{0}} \int_{u_{0}}^{u} h_{i}\left(u^{\prime}\right) \mathrm{d} u^{\prime}
$$

Next, we substitute $u^{\prime}=u_{0}+\alpha$, and then replace $u$ with its first order approximation, which can be found from $u-u_{0} \approx r+z$. The result is

$$
f_{i}(t)=\frac{1}{r+z} \int_{0}^{r+z} h_{i}(c t-r+\alpha) \mathrm{d} \alpha .
$$

Similar calculations can be carried out for the return trip and lead to

$$
T_{2}(t)=T+\frac{1}{2} T\left[\left(a_{x}^{2}-a_{y}^{2}\right) g_{+}(t)+2 a_{x} a_{y} g_{\times}(t)\right],
$$

except that in this case $u-u_{0} \approx r-z$. Therefore,

$$
g_{i}(t)=\frac{1}{r-z} \int_{0}^{r-z} h_{i}(c t-r+z+\beta) \mathrm{d} \beta
$$

The duration of the photon round trip can then be found as

$$
\begin{aligned}
T_{\text {r.t. }}(t) & =T_{1}\left[t-T_{2}(t)\right]+T_{2}(t) \\
& \approx T_{1}(t-T)+T_{2}(t) .
\end{aligned}
$$

It is worthwhile to separate the large unperturbed value from this quantity, and write the result as

$$
T_{\text {r.t. }}(t)=2 T+\delta T_{\text {r.t. }}(t) .
$$

The second term in the right-hand side represents the small variation of the round-trip time which is induced by the gravitational wave,

$$
\delta T_{\text {r.t. }}(t)=\frac{1}{2} T\left[\left(a_{x}^{2}-a_{y}^{2}\right) A_{+}(t)+2 a_{x} a_{y} A_{\times}(t)\right],
$$

where

$$
A_{i}(t)=f_{i}(t-T)+g_{i}(t)
$$

Further calculations become somewhat simpler if we switch to the frequency domain by means of either Fourier or Laplace transformations. 


\subsection{Laplace-domain transfer function}

The Laplace transform of an arbitrary function of time $F(t)$ is given by

$$
\tilde{F}(s)=\int_{0}^{\infty} \mathrm{e}^{-s t} F(t) \mathrm{d} t .
$$

Since $A_{i}$ are linear (integral) transforms of $h_{i}$, we can introduce a transfer function $D(s, \hat{a})$ in the Laplace domain such that

$$
\tilde{A}_{i}(s)=D(s, \hat{a}) \tilde{h}_{i}(s)
$$

for both $i=+$ and $\times$. In this equation we used the following definition:

$$
\tilde{h}_{i}(s)=\int_{0}^{\infty} \mathrm{e}^{-s t} h_{i}(c t) \mathrm{d} t .
$$

Then (41) can be written as

$$
\delta \tilde{T}_{\text {r.t. }}(s)=T D(s, \hat{a})\left[\left(a_{x}^{2}-a_{y}^{2}\right) \tilde{h}_{+}(s)+2 a_{x} a_{y} \tilde{h}_{\times}(s)\right],
$$

where $D(s, \hat{a})$ is the corresponding transfer function:

$$
D(s, \hat{a})=\frac{1}{2 s T}\left[\frac{1-\mathrm{e}^{-\left(1-a_{z}\right) s T}}{1-a_{z}}-\mathrm{e}^{-2 s T} \frac{1-\mathrm{e}^{\left(1+a_{z}\right) s T}}{1+a_{z}}\right] .
$$

The Fourier-domain version of (47) can be obtained by the substitution, $s=2 \pi \mathrm{i} f$, and yields equation (14) in [5]. (For complete equivalence, one must also replace $a_{z}$ with $\hat{a} \cdot \hat{n}$, where $\hat{n}$ is the unit vector in the direction of the source of the gravitational waves.)

\section{Eikonal equation}

A continuous electromagnetic wave with frequency $\omega$ and wavenumber $k(k \equiv \omega / c)$ is described by a scalar function $\phi(x)$ called the eikonal or phase. In this picture, the wavefronts are defined by the surfaces of constant phase and the rays, along which they propagate, are defined by the normals to these surfaces,

$$
p_{\mu}=-\frac{\partial \phi}{\partial x^{\mu}} \text {. }
$$

Consequently, the phase must satisfy the eikonal equation [16]:

$$
g^{\mu \nu} \frac{\partial \phi}{\partial x^{\mu}} \frac{\partial \phi}{\partial x^{\nu}}=0 .
$$

The solution can be found from (48) by direct integration:

$$
\phi=\phi_{0}-\int_{P_{0}}^{P} p_{\mu} \mathrm{d} x^{\mu},
$$

and in general is a function of two points, $P_{0}$ and $P$. Here $\phi_{0}$ is the value of the eikonal at $P_{0}$ which may not necessarily be constant.

If the ray originates at the source of the electro-magnetic waves, the first term in the right-hand side of (50) represents the phase of the source, $\phi_{0} \equiv \phi_{S}\left(t_{0}\right)$, where

$$
\phi_{S}(t)=\omega t \text {. }
$$

The second term in (50) represents the change of the phase along the ray,

$$
\Delta \phi \equiv-\int_{P_{0}}^{P} p_{\mu} \mathrm{d} x^{\mu}
$$


Since three out of four components of the photon momentum are constant along the ray, we readily obtain

$$
\Delta \phi=-p_{x 0} x-p_{y 0} y-p_{v 0}\left(v-v_{0}\right)-\int_{u_{0}}^{u} p_{u}\left(u^{\prime}\right) \mathrm{d} u^{\prime} .
$$

For simplicity, we assumed that the coordinates of $P_{0}$ are $x_{0}=y_{0}=z_{0}=0$. Furthermore, the last two terms on the right-hand side of (52) are equal. This can be seen from (21) by changing the integration variable $\sigma$ to $u$ :

$$
v=v_{0}+\frac{1}{p_{v 0}} \int_{u_{0}}^{u} p_{u}\left(u^{\prime}\right) \mathrm{d} u^{\prime}
$$

Knowing this, we can write the phase change along the ray as

$$
\Delta \phi=-p_{x 0} x-p_{y 0} y-2 p_{v 0}\left(v-v_{0}\right) .
$$

In the boundary-value problem considered in section 3.1, $p_{\mu}$ are functions of the coordinates of the end point, (23) 25). Substituting these formulae into (55), we obtain

$$
\Delta \phi\left(t_{0}, t\right)=\frac{1}{\sigma}\left[c^{2}\left(t-t_{0}\right)^{2}-r^{2}-\left(x^{2}+y^{2}\right) f_{+}(t)-2 x y f_{\times}(t)\right],
$$

where we show explicitly its dual time dependence. Note that if we follow a surface of constant phase, the two points $P$ and $P_{0}$ will correspond to the same wavefront and therefore $\Delta \phi_{1}=0$. In this case, (56) will yield the photon propagation time (27).

\section{Round-trip phase}

Consider now a situation when the wave is reflected at some point, say $P_{1}$, and from then on proceeds along a different ray. Let the end point along the new ray be $P$. Since the eikonal is an additive quantity, its value at the end point is

$$
\phi(t)=\phi_{0}\left(t_{0}\right)+\Delta \phi_{1}\left(t_{0}, t_{1}\right)+\Delta \phi_{2}\left(t_{1}, t\right) .
$$

Here $\Delta \phi_{1}$ is given by (56) with $t_{1}$ replacing $t$ and

$$
\Delta \phi_{2}=-\int_{P_{1}}^{P} q_{\mu} \mathrm{d} x^{\mu}
$$

where $q_{\mu}$ is the photon momentum along the second ray. Obviously, this approach can be extended to an arbitrary number of reflections.

We can now apply (57) to find the phase of the wave returning to the source after one round trip. In this case $q_{\mu}$ are given by (28) 30 . Sustituting these formulae into (58), we obtain the phase increment for the return trip,

$$
\Delta \phi_{2}\left(t_{1}, t\right)=\frac{1}{\sigma}\left[c^{2}\left(t-t_{1}\right)^{2}-r^{2}-\left(x^{2}+y^{2}\right) g_{+}(t)-2 x y g_{\times}(t)\right] .
$$

In this case too, the condition for constant phase along the ray $\left(\Delta \phi_{2}=0\right)$ yields the photon propagation time (31).

Following a particular wave along the round trip, we find that both phase increments $\Delta \phi_{1}$ and $\Delta \phi_{2}$ vanish and that the phase at the end of the round trip is

$$
\phi(t)=\phi_{S}\left(t_{0}\right)=\omega t_{0},
$$

in which $t_{0}$ lags $t$ by the duration of one round trip:

$$
\phi(t)=\omega\left[t-T_{\text {r.t. }}(t)\right] .
$$




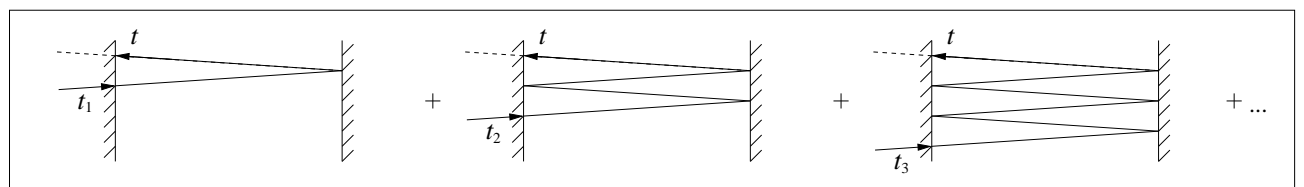

Figure 1. Schematic representation of the superposition of fields in a Fabry-Perot resonator. At any given time, $t$, the complex amplitude of the wave inside the resonator can be viewed as a sum of the complex amplitudes of an infinite number of waves. Each wave corresponds to a ray which completed a certain number of round trips: $1,2,3, \ldots, n$. The rays enter the resonator at times $t_{1}, t_{2}, t_{3}, \ldots t_{n}$.

Thus, the phase difference between the wave returning to the source and the wave presently emmited by the source is

$$
\phi(t)-\phi_{S}(t)=-\omega T_{\text {r.t. }}(t)=-2 k L-\psi(t),
$$

where $k L=\omega T$ is the large unperturbed value of the phase, and

$$
\psi(t)=\omega \delta T_{\text {r.t. }}(t)
$$

is the small phase shift due to the presence of the gravitational wave. We have thus proved equation (15) in $[5]$.

\section{Light storage in a Fabry-Perot resonator}

\subsection{Superposition of fields}

The phase shift acquired by the light during one round trip can be amplified by means of multiple reflections. One way of doing so is to store the light in a Fabry-Perot resonator which consists of two mirrors separated by a distance $L$ from each other. The analysis of single wave propagation in sections 4 and 5 can be extended to multiple waves using the principle of superposition. Namely, the wave inside a Fabry-Perot resonator can be viewed as a superposition of waves corresponding to rays which have undergone multiple reflections inside the resonator as shown in figure 1.

Consider a wave incident on a Fabry-Perot resonator and assume that its amplitude is constant in time, $A_{0}$. As we know, the phase of the wave will evolve according to the eikonal equation (49) and in general will be a function of position and time. In what follows, all quantities will be considered at a fixed location (the front mirror of the resonator) and therefore will be functions of time only. Then the complex amplitude of the wave incident on the front mirror of the resonator can be written as $\mathcal{E}_{\text {in }}(t)=A_{0} \mathrm{e}^{\mathrm{i} \phi(t)}$. The complex amplitude of the electromagnetic wave inside the Fabry-Perot resonator can be found by superposition:

$$
\mathcal{E}(t) \equiv \sum_{n=1}^{\infty} \mathcal{E}_{n}(t)=\sum_{n=1}^{\infty} A_{n} \mathrm{e}^{\mathrm{i} \phi_{n}(t)}
$$

where $A_{n}$ and $\phi_{n}(t)$ are the amplitude and phase of the wave, which corresponds to the $n$-th ray inside the resonator. Let the reflectivity of the mirrors be $r$ and $r^{\prime}$ and the transmissivity of the front mirror be $\tau$. Then $A_{1}=A_{0} \tau\left(-r^{\prime}\right)$ and

$$
A_{n}=A_{1}\left(r r^{\prime}\right)^{n-1}=-\frac{\tau}{r} A_{0}\left(r r^{\prime}\right)^{n} .
$$


(Here we assumed the usual sign reversal upon reflection.) The phases which correspond to the individual rays can be found from (61). Namely, the phase of the first ray is

$$
\phi_{1}(t)=\phi_{S}\left(t_{1}\right), \quad \text { where } \quad t_{1}=t-T_{\text {r.t. }}(t),
$$

the phase of the second ray is

$$
\phi_{2}(t)=\phi_{S}\left(t_{2}\right), \quad \text { where } t_{2}=t_{1}-T_{\text {r.t. }}\left(t_{1}\right),
$$

and so on. The general formula for the phase is

$$
\phi_{n}(t)=\phi_{S}\left(t_{n}\right), \quad \text { where } \quad t_{n}=t-\sum_{m=0}^{n-1} T_{\text {r.t. }}\left(t_{m}\right),
$$

for $n=1,2,3, \ldots$ Separating $T_{\text {r.t. }}(t)$ into two components, as in (40), and neglecting the terms of order $h$ in the argument of $\delta T_{\text {r.t. }}\left(t_{m}\right)$, we obtain $t_{n}$ in closed form:

$$
t_{n}=t-2 n T-\sum_{m=0}^{n-1} \delta T_{\text {r.t. }}(t-2 m T),
$$

where $T \equiv L / c$. The corresponding phase can then be written as

$$
\phi_{n}(t) \equiv \omega t_{n}=\omega t-2 n k L-\sum_{m=0}^{n-1} \psi(t-2 m T),
$$

where $\psi$ is the phase induced by the gravitational wave in one round trip (63). Substituting (70) into (64), we obtain

$$
\mathcal{E}(t)=\sum_{n=1}^{\infty} A_{n} \mathrm{e}^{\mathrm{i} \omega t} \exp \left[-2 \mathrm{i} n k L-\mathrm{i} \sum_{m=0}^{n-1} \psi(t-2 m T)\right] .
$$

This formula describes the superposition of an infinite series of electromagnetic waves in a Fabry-Perot resonator which is placed in the field of a weak gravitational wave. The phases $\psi$ are determined by the propagation times of the associated photon round trips (63).

\subsection{Amplification of phase}

We can now identify the amplitude and the phase of the internal field from (71). First, we notice that $A_{n}$ contains $\left(r r^{\prime}\right)^{n}$ which can be combined with $\exp (-2 \mathrm{i} n k L)$ to yield $\rho^{n}$, where $\rho=r r^{\prime} \exp (-2 \mathrm{i} k L)$. Second, we notice that $\psi$ is very small (on the order of $h$ ) and therefore we can approximate: $\mathrm{e}^{-\mathrm{i} \psi} \approx 1-\mathrm{i} \psi$. With these changes, (71) becomes

$$
\mathcal{E}(t)=-\frac{\tau}{r} A_{0} \mathrm{e}^{\mathrm{i} \omega t}\left(\sum_{n=1}^{\infty} \rho^{n}-\mathrm{i} \sum_{n=1}^{\infty} \sum_{m=0}^{n-1} \gamma_{n m}\right),
$$

where we introduced the temporary notation: $\gamma_{n m}=\rho^{n} \psi(t-2 m T)$. The first sum in (72) is a geometric progression which amounts to $\rho /(1-\rho)$. The second (double) sum in (72) can be simplified by resummation:

$$
\sum_{n=1}^{\infty} \sum_{m=0}^{n-1} \gamma_{n m}=\sum_{m=0}^{\infty} \sum_{n=m+1}^{\infty} \gamma_{n m}=\frac{\rho}{1-\rho} \sum_{m=0}^{\infty} \rho^{m} \psi(t-2 m T) .
$$

$\S$ Whenever $t_{0}$ occurs in this series it should be replaced with $t$. 
With these changes, (72) becomes

$$
\mathcal{E}(t)=-\frac{\tau}{r} A_{0} \mathrm{e}^{\mathrm{i} \omega t} \frac{\rho}{1-\rho}\left[1-\mathrm{i} \sum_{m=0}^{\infty} \rho^{m} \psi(t-2 m T)\right] .
$$

To first order in $h$, the expression in the square brackets can be approximated by a phase factor $\mathrm{e}^{-\mathrm{i} \Psi(t)}$. Therefore,

$$
\mathcal{E}(t)=A \mathrm{e}^{\mathrm{i} \omega t-\mathrm{i} \Psi(t)},
$$

where $A$ is the unperturbed amplitude of the field and $\Psi(t)$ is the phase shift due to the gravitational wave. In the explicit form,

$$
A=-\frac{\tau \rho}{r(1-\rho)} A_{0}
$$

and

$$
\Psi(t)=\sum_{m=0}^{\infty} \rho^{m} \psi(t-2 m T) .
$$

The Laplace-domain version of (77) can be written as

$$
\tilde{\Psi}(s)=g_{0} C(s) \tilde{\psi}(s), \quad C(s)=\frac{1-\rho}{1-\rho \mathrm{e}^{-2 s T}},
$$

where $g_{0}=(1-\rho)^{-1}$ is the amplification factor and $C(s)$ is the transfer function of a Fabry-Perot resonator normalized to 1 at $s=0$. In a perfectly tuned Fabry-Perot resonator, the length $L$ is equal to an integer number of laser half-wavelengths and therefore $\mathrm{e}^{-2 \mathrm{i} k L}=1$ and $\rho=r r^{\prime}$. We have thus derived equations (26) and (27) in 5. Note that the 4 -km LIGO interferometers have gain $g_{0} \approx 70$ which amounts to a 70 -fold increase in the sensitivity of these detectors from the Fabry-Perot effect.

\section{Response of detectors to gravitational waves}

We conclude with a brief derivation of the detector response functions for two widely known configurations: a simple Michelson interferometer and a Michelson interferometer with Fabry-Perot arm cavities. For a schematic diagram of these detectors and definitions of the field amplitudes see figure 2 in $[5$.

In a simple Michelson configuration, the signal is produced by interfering two beams which return to the beam splitter after completing a round trip in the interferometer arms. Denote the phases induced by the gravitational wave in each arm by $\psi(t, \hat{a})$ and $\psi(t, \hat{b})$, where $\hat{a}$ and $\hat{b}$ are the unit vectors along the interferometer arms. Once the unit vectors are defined, the phases can be found from (63) and (411). If the beam splitter is set to the dark fringe, the signal is proportional to the difference in the phases of the two beams. With appropriate normalization [5], it is given by

$$
V(t)=\frac{1}{2 \omega T}[\psi(t, \hat{a})-\psi(t, \hat{b})] .
$$

Taking the Laplace transformation and substituting for the phases from (63) and (46), we obtain

$$
\tilde{V}(s)=G_{+}(s) \tilde{h}_{+}(s)+G_{\times}(s) \tilde{h}_{\times}(s)
$$


where $G_{i}(s)$ are the transfer functions for the two polarizations of the gravitational wave:

$$
\begin{aligned}
& G_{+}(s)=\frac{1}{2}\left(a_{x}^{2}-a_{y}^{2}\right) D(s, \hat{a})-\frac{1}{2}\left(b_{x}^{2}-b_{y}^{2}\right) D(s, \hat{b}), \\
& G_{\times}(s)=a_{x} a_{y} D(s, \hat{a})-b_{x} b_{y} D(s, \hat{b}) .
\end{aligned}
$$

Equations (81) and (82) are equivalent to equation (19) in [5].

Similarly, in a Michelson configuration with Fabry-Perot resonators in the interferometer arms, the signal is given by

$$
V(t)=\frac{1}{2 \omega T}[\Psi(t, \hat{a})-\Psi(t, \hat{b})],
$$

where the phases $\Psi(t, \hat{a})$ and $\Psi(t, \hat{b})$ correspond to the internal fields in each resonator arm. They can be found from (77). In the Laplace domain, the signal can be written as

$$
\tilde{V}(s)=g_{0}\left[H_{+}(s) \tilde{h}_{+}(s)+H_{\times}(s) \tilde{h}_{\times}(s)\right] .
$$

The corresponding transfer functions are

$$
H_{i}(s)=C(s) G_{i}(s) \text {, }
$$

where $C(s)$ is given by (78) and $G_{i}(s)$ are given by (81) and (82). We have thus derived equations (30) and (31) in [5]. (For normalization purpose, the factor $g_{0}$ was ommitted in [5].)

\section{Conclusion}

We have seen that the response of laser interferometers to gravitational waves derived within the framework of general relativity agrees with that of the traditional semirigorous approach. The present analysis provides a rigorous derivation of the key formulae in [5] which constitute an accurate model for the detector response valid at all frequencies. Such an accurate model is required for analysis of the detector's sensitivity at high frequencies where the conventional long-wavelength approximation breaks down. This includes recent studies of the possibility of searches for gravitational waves at the free spectral range $(37.5 \mathrm{kHz})$ of the 4 -km LIGO interferometers [17, 18, where the sensitivity peaks in a narrow $(\sim 200-\mathrm{Hz})$ band. The increased sensitivity of the detectors at this frequency led to the development of new data-analysis efforts targeting searches for high-frequency burst [19] and stochastic [20] gravitational waves.

\section{Acknowledgments}

The author acknowledges the support from the Center for Gravitational Wave Physics at the Pennsylvania State University and the University of Texas at Brownsville. This work was supported by the US National Science Foundation under grant PHY 0734800. This paper has been assigned LIGO Document Number P070060 and was circulated for the internal review by the LIGO Scientific Collaboration on Oct. 23, 2008. 
Photon round trips in the field of a plane gravitational wave

\section{References}

[1] Barish B and Weiss R 1999 Phys. Today 5244

[2] Bradaschia C et al. 1990 Nucl. Instrum. Methods 289518

[3] Thorne K 1987 in S Hawking and W Israel, eds, 300 Years of Gravitation (Cambridge University Press)

[4] Baskaran D and Grishchuk L 2004 Class. Quantum Grav. 214041

[5] Rakhmanov M, Romano J and Whelan J 2008 Class. Quantum Grav. 25184017

[6] Gürsel Y, Linsay P, Spero R, Saulson P, Whitcomb S and Weiss R 1984 in A Study of a Long Baseline Gravitational Wave Antenna System (National Science Foundation Report)

[7] Christensen N 1992 Phys. Rev. D 465250

[8] Sigg D 1997 Strain calibration in LIGO. LIGO Technical Report T970101-B

[9] Schilling R 1997 Class. Quantum Grav. 141513

[10] Larson S, Hiscock W and Hellings R 2000 Phys. Rev. D 62062001

[11] Cornish N and Larson S 2001 Class. Quantum Grav. 183473

[12] Cornish N and Rubbo L 2003 Phys. Rev. D 67022001

[13] Whelan J 2007 Higher-frequency corrections to stochastic formulae. LIGO Technical Report T070172

[14] Rakhmanov M 2006 Response of LIGO 4-km interferometers to gravitational waves at high frequencies and in the vicinity of the FSR $(37.5 \mathrm{kHz})$. LIGO Technical Report T060237

[15] Misner C, Thorne K and Wheeler J 1973 Gravitation (San Francisco: W.H. Freeman and Company)

[16] Landau L and Lifshitz E 1971 The Classical Theory of Fields (New York: Pergamon Press)

[17] Sigg D and Savage R 2003 Analysis proposal to search for gravitational waves at multiples of the LIGO arm cavity free-spectral-range frequency. LIGO Technical Report T030296

[18] Hunter E 2005 Analysis of the frequency dependence of the LIGO directional sensitivity (antenna pattern) and implications for detector calibration. LIGO Technical Report T050136

[19] Parker J 2007 Development of a high-frequency burst analysis pipeline. LIGO Technical Report T070037

[20] Giampanis S 2008 Search for a high frequency stochastic background of gravitational waves $P h D$ Thesis University of Rochester 